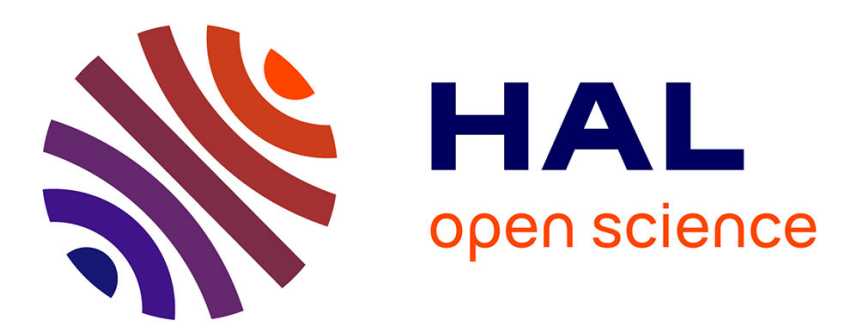

\title{
Reynolds dependence of third-order velocity structure functions
}

Yves Gagne, Bernard Castaing, Christophe Baudet, Yann Malecot

\section{To cite this version:}

Yves Gagne, Bernard Castaing, Christophe Baudet, Yann Malecot. Reynolds dependence of thirdorder velocity structure functions. Physics of Fluids, 2004, 16 (2), pp.482-485. 10.1063/1.1639013 . hal-00183628

\section{HAL Id: hal-00183628 \\ https://hal.science/hal-00183628}

Submitted on 5 Feb 2020

HAL is a multi-disciplinary open access archive for the deposit and dissemination of scientific research documents, whether they are published or not. The documents may come from teaching and research institutions in France or abroad, or from public or private research centers.
L'archive ouverte pluridisciplinaire HAL, est destinée au dépôt et à la diffusion de documents scientifiques de niveau recherche, publiés ou non, émanant des établissements d'enseignement et de recherche français ou étrangers, des laboratoires publics ou privés. 


\title{
Reynolds dependence of third-order velocity structure functions
}

\author{
Yves Gagne \\ Institut de Mécanique de Grenoble, LEGI, UJF/INPG/CNRS, BP 53, 38041-Grenoble-Cedex 9, France \\ Bernard Castaing \\ ENSL/CNRS, 44 Alle d'Italie, 69364 Lyon-Cedex 7, France \\ Christophe Baudet and Yann Malécot \\ Institut de Mécanique de Grenoble, LEGI, UJF/INPG/CNRS, BP 53, 38041-Grenoble-Cedex 9, France
}

(Received 25 March 2003; accepted 24 October 2003; published online 8 January 2004)

We study the experimental dependence of the third-order velocity structure function on the Taylor based Reynolds number, obtained in different flow types over the range $72 \leqslant R_{\lambda} \leqslant 2260$. As expected, when the Reynolds number is increasing, the third-order velocity structure functions (plotted in a compensated way) converge very slowly to a possible $-4 / 5$ plateau value according to the Kolmogorov 41 theory. Actually, each of these normalized third-order functions exhibits a maximum, at a scale close to the Taylor microscale $\lambda$. In this Brief Communication, we show that experimental data are in good agreement with the recent predictions of Qian and Lundgren. We also suggest that, from an experimental point of view, a log-similar plot suits very well to study carefully the behavior of the third-order velocity structure functions with the flow Reynolds number. (C) 2004 American Institute of Physics. [DOI: 10.1063/1.1639013]

Considerable attention has been given to the famous “- $4 / 5$ " law, ${ }^{1,2}$ characteristic of inertial scales in fully developed turbulence which is written as

$$
\left\langle(\delta u(r))^{3}\right\rangle \simeq-4 / 5\langle\epsilon\rangle r
$$

( $r$ and $\epsilon$ are the inertial range separation and the mean dissipation rate, respectively). Even though the previous relation is strictly valid for Reynolds number tending to the infinity, experimental data seemed to verify it as soon as a conspicuous power-law scaling range exists (i.e., for $R_{\lambda} \geqslant 500$ ), whatever the flow type. To emphasize this feature, the third-order longitudinal velocity structure functions are usually compensated by the opposite of the Kolmogorov scaling term [defined as $-\left\langle(\delta u(r))^{3}\right\rangle /(\langle\epsilon\rangle r)$ and hereafter noted $\left.S_{3}(r)\right]$, and are plotted in $\log -\log$ coordinates (cf. Refs. 3, 4, and references therein). Actually, the difference between $S_{3}(r)$ and $4 / 5$ is experimentally very difficult to determine. Indeed, the identification of $S_{3}(r)$ with $4 / 5$ is very often the most accurate way to experimentally determine $\langle\epsilon\rangle$ at large $R_{\lambda}$.

However, some experiments have shown a very slow convergence towards the above infinite Reynolds result when $R_{\lambda}$ is raised. For instance, in a "Von Karman" flow, Moisy" showed that $S_{3}(r)$ tends to $4 / 5$ like $R_{\lambda}^{-6 / 5}$. By another way, Mydlarski and Warhaft, ${ }^{6}$ found a slow convergence of the slope of the energy spectrum measured in a grid turbulence towards $-5 / 3$ as $R_{\lambda}$ increases. For the peculiar scale $r=\lambda$, Pearson and Antonia ${ }^{7}$ found that $S_{3}(\lambda)$ does not exactly scale as the Kolmogorov prediction for Reynolds number up to $R_{\lambda} \simeq 1000$, reflecting the large scale anisotropy effects on the inertial range.

On the theoretical side, in some recent papers, ${ }^{8,9}$ Qian examines this problem, dicussing systematically the influence of the energy input. Qian predicts the slowness of the convergence, but points out noticeable differences between various types of power injection. Lundgren ${ }^{10,11}$ goes back to the Karman-Howarth equation to propose a solution in the decaying self-similar case which seems not easily compatible with the possibility of intermittency.

In the present state, it is difficult to decide if an experimental discrepancy with the Lundgren prediction is due to (i) a peculiar injection regime as discussed by Qian, (ii) an intermittency effect, (iii) or, simply, an experimental error in the determination of $R_{\lambda}$. In this Brief Communication, we show that a suitable diagram allows to illustrate how the various theoretical predictions overlap, and to decide among the above questions.

Let us summarize the Qian and Lundgren results. Based on the additive property of the energy input and dissipation, the main prediction of Qian [Eq. (24) in Ref. 9], valid for $\eta \ll r \ll \ell_{0}$, is

$$
S_{3}(r)=4 / 5-C_{1}\left(r / \ell_{0}\right)^{m}-C_{2}(r / \eta)^{-4 / 3}
$$

with $C_{1}>0$ and $C_{2} \simeq 5.26 K_{0}$ where $K_{0}$ is the Kolmogorov constant, $\ell_{0}$ the large scale, and $\eta$ the viscous scale. The second term of the right-hand side represents the large-scale effect and the last term the viscosity effect. At the particular scale where $S_{3}(r)$ is maximum, the above relation leads to

$$
\delta=4 / 5-\left[S_{3}(r)\right]_{\max }=C_{\delta} R_{\lambda}^{-\mu}, \quad \mu=\frac{6 m}{(3 m+4)} .
$$

From this Reynolds dependence of $\left[S_{3}(r)\right]_{\max }$, Qian has deduced several predictions of the exponent $\mu$ depending on the types of flow. For example, he found $2 / 3 \leqslant \mu \leqslant 1$ in the homogeneous shear-flow turbulence. For some types of large-scale forcing turbulence, he predicted an exponent equal to $\mu=6 / 5$, in agreement with the Moisy experiments. ${ }^{5}$ In the interesting case of a decaying self-preserving turbu- 
TABLE I. The basic parameters of the data sets.

\begin{tabular}{llcccc}
\hline \hline & \multicolumn{2}{c}{ Grid turbulence } & \multicolumn{2}{c}{ Jet } & ONERA \\
\hline$R_{\lambda}$ & 72 & 144 & 350 & 695 & 2260 \\
$U(\mathrm{~m} / \mathrm{s})$ & 4.95 & 19.6 & 1.68 & 6.3 & 20.75 \\
$u^{\prime}(\mathrm{m} / \mathrm{s})$ & 0.17 & 0.64 & 0.44 & 1.55 & 1.58 \\
$\eta(\mathrm{mm})$ & 0.4 & 0.15 & 0.33 & 0.14 & 0.30 \\
$\lambda(\mathrm{cm})$ & 1.02 & 0.35 & 1.23 & 0.67 & 2.80 \\
$L(\mathrm{~cm})$ & 4 & 4 & 20 & 20 & $\simeq 410$ \\
$\langle\epsilon\rangle\left(\mathrm{m}^{2} / \mathrm{s}^{3}\right)$ & 0.0093 & 7.7 & 0.29 & 11.7 & 0.95 \\
$10^{5} \nu\left(\mathrm{m}^{2} / \mathrm{s}\right)$ & 1.51 & 1.55 & 1.52 & 1.53 & 2 \\
\hline \hline
\end{tabular}

lence, Qian's prediction is $\mu=2 / 3$. In this case, Lundgren has also derived an equation for $S_{3}(r)$ [Eq. (45) of Ref. 10] by using a method of matched asymptotic expansions. In terms of $\eta$, it can be written as

$$
S_{3}(r)=4 / 5-\frac{3.34}{\sqrt{15}}\left(r / \ell_{0}\right)^{2 / 3}-8(r / \eta)^{-4 / 3}
$$

which leads to

$$
\left[S_{3}(r)\right]_{\max }=4 / 5-8.45 R_{\lambda}^{-2 / 3} .
$$

Note that the coefficients of Eqs. (4) and (5) are compatible with those of Eqs. (2) and (3) when $K_{0}=1.5-1.8$ (see Table II of Ref. 9).

In order to test these theoretical predictions, we have used three different types of air flows: a round jet, a grid turbulence and in the return channel flow of the ONERAModane wind tunnel. In the laboratory flows, measurements of the longitudinal velocity fluctuations were performed in classical conditions, respectively, on the axis of the jet at $x / d=38$ downstream the nozzle (with a diameter $d$ $=12 \mathrm{~cm}$ ), and at $x / M=40$ downstream a square bars grid (with a mesh size of $M=4 \mathrm{~cm}$ and a solidity of 0.36 ). At Modane, the probe was located on the axis of the $24 \mathrm{~m}$ diameter return channel. In such an industrial facility, the flow generates a "chunk" turbulence. Further details on these experimental conditions are given in Ref. 12. Velocity signals were obtained using the CTA hot wire technique with a home made single-wire probe. The hot wires used in the laboratory flows and in the industrial flow, were $0.3 \mathrm{~mm}$ and $0.5 \mathrm{~mm}$ in length and $2.5 \mu \mathrm{m}$ and $3.5 \mu \mathrm{m}$ in diameter, respectively, and were operated on DISA 55M01 anemometers. The real time duration of data records (of about 75 megasamples) was always greater than 12000 times the integral time scales. The time-space conversion of signals has carried out a "corrected Taylor hypothesis" using the instantaneous velocity. ${ }^{13,14}$ Table I lists the relevant data for the records analyzed here, estimated with the usual homogeneous and isotopic definitions: $\langle\epsilon\rangle=15 \nu\left\langle u^{2}\right\rangle / \lambda^{2}, \quad \eta$ $=\left(\nu^{3} /\langle\epsilon\rangle\right)^{1 / 4}, \lambda^{2}=\left\langle u^{2}\right\rangle /\left\langle(\partial u / \partial x)^{2}\right\rangle$.

Figure 1 shows compensated third-order longitudinal structure functions $S_{3}(r)$ plotted against the separation distance $r$ in linear coordinates. In addition, we have reported the data obtained from an atmospheric turbulence, that we have extracted by hand from Fig. 1 of the paper of Dhruva et al. ${ }^{15}$ Following the authors, $R_{\lambda}$ is about $10^{4}-1,4 \times 10^{4}$. In Fig. 1, no plateau is observed, the third-order velocity

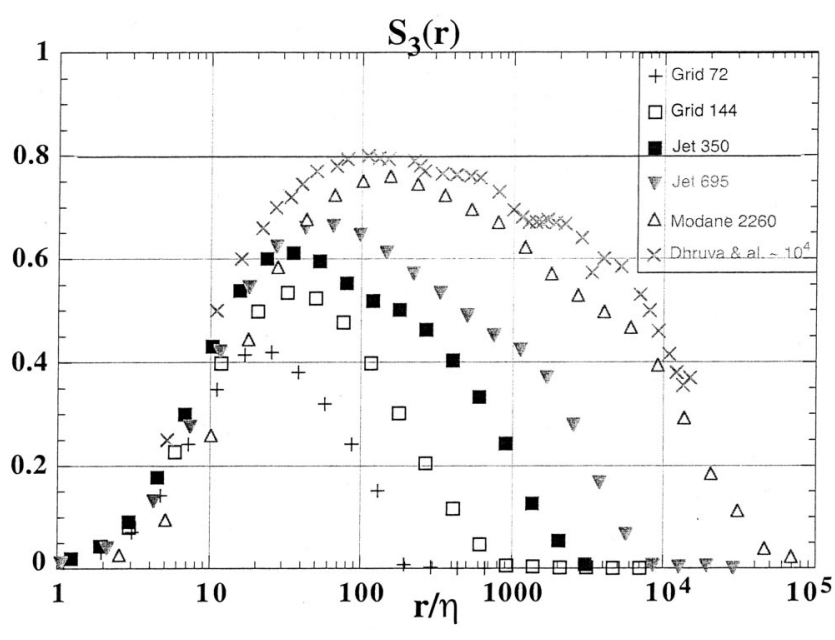

FIG. 1. $S_{3}(r)$ in lin-log coordinates.

structure function does not strictly behave as a power law, even in the middle of the inertial range of one of the highest Reynolds number flow never investigated. Note that the accuracy of measurements does not affect the shapes of the $S_{3}(r)$ (and cannot lessen this finding), it only affects the level value of the $S_{3}(r)$ which drastically depends on the squares of the velocity gradients calculated at the Kolmogorov scale $\eta$. As expected, these maxima continuously converge to the horizontal line $4 / 5$ as $R_{\lambda}$ is increasing. This monotonous evolution suggests that data have been obtained with a rather good accuracy despite the difficulty to measure the small scale velocity gradients. The lack of a true " $-4 / 5$ plateau" has been studied in detail by Danailla et al. ${ }^{16}$ in the case of a grid turbulence. From the complete form of the Kolmogorov equation involving both the viscous and the nonstationary terms, this Brief Communication shows how the latter term acts as a negative production term at large scale. The authors showed that experimental data verify the Kolmogorov equation if one accounts for the nonstationarity of the second-order moments. The aim here is not to study this interesting finding in others types of flow, but rather to compare behaviors of the functions $S_{3}(r)$ towards the flow type and the Reynolds number value.

A way to highlight the gap between $\left[S_{3}(r)\right]_{\max }$ and $4 / 5$ studied by Qian ${ }^{8,9}$ and Lundgren, ${ }^{10,11}$ is to plot the quantity $\left(1-\left(5 S_{3}(r) / 4\right)\right) R_{\lambda}^{2 / 3}$ versus the separation $r$. Such a stringent diagram is shown on Fig. 2, where the separation $r$ has been rescaled with the Taylor microscale $\lambda$ (and not with $\eta$ as in Fig. 1). We observe (as in Ref. 5) that all the minima of the experimental curves (corresponding to $\left[S_{3}(r)\right]_{\max }$ ) occur at a unique scale close to $\lambda$. To our knowledge, there is no physical explanation of this feature. Even though the minima values agree (more or less) with the previous theoretical predictions $[\ln (8.45)=2.13]$, these curves diverge each other both at large and small scales.

In fact, due to the existence of the two scales $\ell_{0}$ and $\eta$, the problem is that neither $r / \ell_{0}$ nor $r / \eta$ can be considered as the good variable. A characteristic scale in turbulence is not identified by its ratio to $\ell_{0}$ but by the way this ratio scales with the Reynolds number. If this scale is almost equal to the 


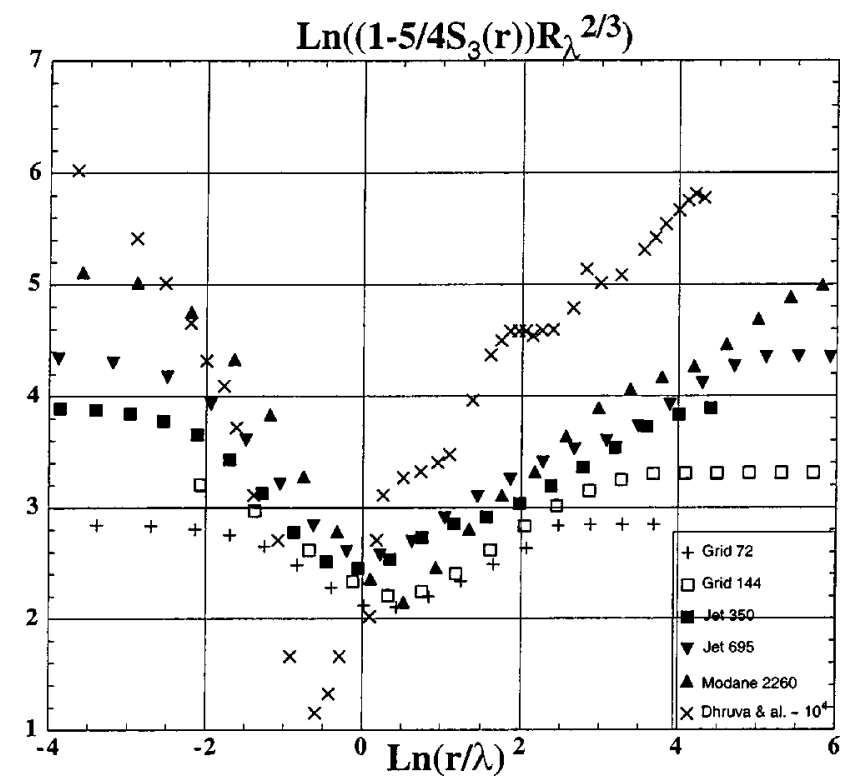

FIG. 2. Compensated $S_{3}(r)$ according to the Qian and Lundgren predictions.

Taylor scale $\lambda$, while its ratio with $\ell_{0}$ scales like $R_{\lambda}^{-3 / 2}$, it must be identified with $\eta$ and not $\lambda$. In this context, as recognized in previous studies, ${ }^{12,17}$ the good variable is of kind $\ln \left(r / \ell_{0}\right) / \ln R_{\lambda}$, in such a way that characteristic scales having the same behavior with $R_{\lambda}$ converge towards the same abcissa when $R_{\lambda}$ tends to infinity. Faster convergence is obtained using $\ln \left(R_{\lambda} / R_{*}\right)$ with $R_{*}=28,{ }^{12,17}$ which ensures that $\ln \left(\ell_{0} / \eta\right)$ remains close to $\frac{3}{2} \ln \left(R_{\lambda} / R_{*}\right)$ down to moderate $R_{\lambda}$. We then propose such a plot. As we want to emphasize the role of $\lambda$, we take the abcissa $\beta \ln (r / \lambda)$ with $\beta$ $=1 / \ln \left(R_{\lambda} / R_{*}\right)$. To maintain the scaling laws, we have to divide the logarithmic ordinates by the same factor $\ln \left(R_{\lambda} / R_{*}\right)$. We thus take as the ordinate $\beta \ln \left(1-\left(5 S_{3}(r) / 4\right)\right)$. In such coordinates, Fig. 3 shows the experimental curves (the same

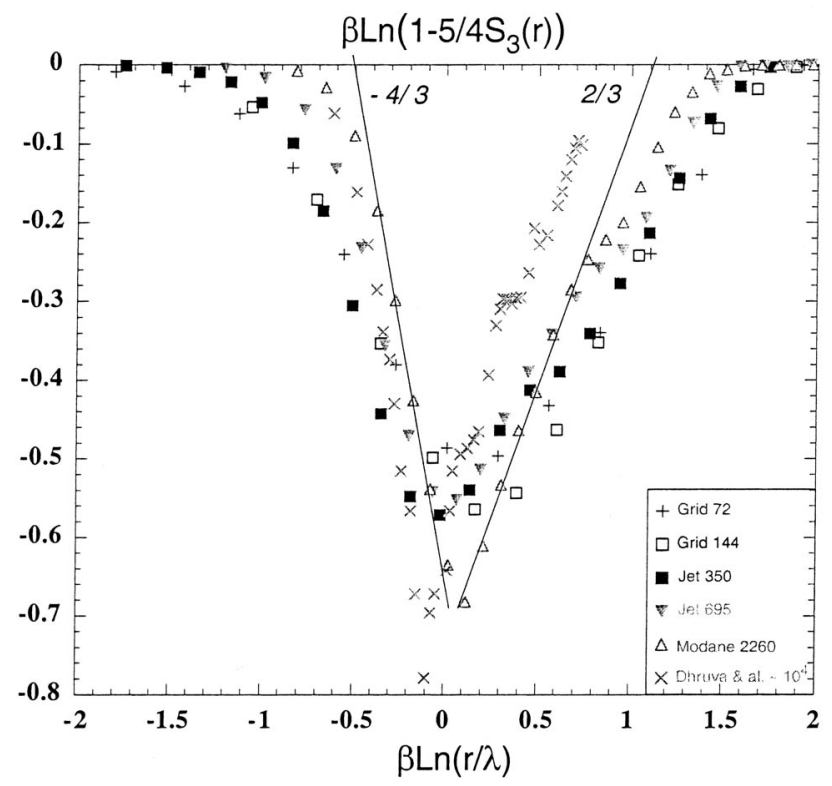

FIG. 3. Compensated $S_{3}(r)$ according to the log-similar plot.

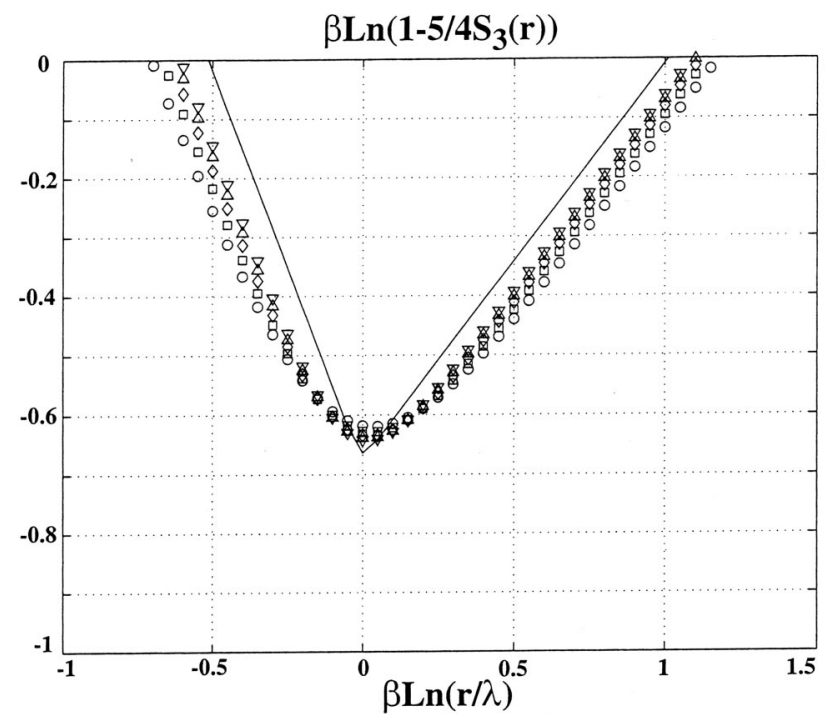

FIG. 4. Lundgren results for $R_{\lambda}=500, \bigcirc ; 1000, \square ; 2000, \diamond ; 4000, \triangle$; $8000, \nabla$; and $\infty$, continuous line.

as those of Fig. 2), while Fig. 4 displays the results obtained from Eq. (4).

While none of the two plots show perfect merging, this presentation has numerous advantages. First, the ordinate $-\mu$ of the minimum gives the Reynolds dependence of the maximum of $S_{3}(r)$ that we define as $\left[S_{3}(r)\right]_{\max }=4 / 5$ $-A\left(R_{\lambda} / R_{*}\right)^{-\mu}$, analogously to the predicted Eqs. (3) and (5). Second, the slopes close to zero ordinate give the power law dependences of the outer scale $\left(\sim \ell_{0}\right)$ and inner scale $(\sim \eta)$ corrections to the $-4 / 5$ law as expressed in Eqs. (2) and (4). Last, the distance between the two points of intersection of each curve with the zero ordinate is bounded by $R_{\lambda}\left(\simeq 3 / 2+\left[3 / \ln \left(R_{\lambda} / 28\right)\right]\right)$. The different data converge towards the infinite Reynolds curve is characteristic of the coefficients of these laws. For instance, it can be seen that choosing $R_{*}=28$ gives $A$ close to 1, both for Eqs. (3) and (5) and for real data. Indeed, this choice comes from an independent work (cf. Ref. 12) and this coincidence has to be remarked. Figure 4 shows how curves degenerate in two straight lines when $R_{\lambda} \rightarrow \infty$. The position of the maximum of $S_{3}$, its dependence with $R_{\lambda}$, the power law dependences of the correction to $S_{3}$, and even their coefficients, all are probably linked. For instance, if we write, as Lundgren did 1 $-\left(5 S_{3} / 4\right)=\left(R_{\lambda} / R_{*}\right)^{-\alpha}\left(A_{0}(r / \lambda)^{\gamma_{0}}+A_{i}(r / \lambda)^{\gamma_{i}}\right)$, we must have $\gamma_{0}-\gamma_{i}=2$ as one of the terms comes from the integral of $\left\langle\delta u(r)^{2}\right\rangle$, and the second from its derivative. Then, to have the maximum $\left[S_{3}(r)\right]_{\max }$ at an abcissa $r$ close to $\lambda$, even at moderate $R_{\lambda}$, we must have $A_{0}=2 / 3$ and $A_{i}=1 / 3$ if $\gamma_{i}=-4 / 3$.

Further remarks can be discussed in relation to the questions addressed in the beginning of this paper.

The two slopes plotted in Fig. 3 (equal to $-4 / 3$ and 2/3 according to the theoretical predictions) seem to be reached only for $R_{\lambda}>1000$ (note that an error on $\beta$ does not affect these slopes). On the large scale side, the two highest Reynolds data behave as nearly as $\sim R^{2 / 3}$ even though they correspond to two very different flows. Moreover, on the large 
scale side of experimental curves, we observe that the correction slope [noted $m$ in Eq. (2)] never exceeds the value $2 / 3$, whatever the flow type. In fact, the different power input types (grid turbulence, jet) are hardly noticeable, contrary to the theoretical expectations.

With regard to the accuracy of the Reynolds number determination, such coordinates clearly reveal that in Ref. 15 data, $R_{\lambda}$ has been grossly overestimated. This underestimates $\beta$ and causes the diagram to shrink.

Other differences between the theoretical predictions and the real data are within the experimental uncertainty. This was to be expected, in particular, the intermittency effect cannot probably be detected in such a diagram, as the following calculus shows.

In his matched asymptotic expansion, Lundgren derives an outer and an inner expression for the second moment $\left\langle\delta u(r)^{2}\right\rangle\left(R_{L}=R_{\lambda}^{2} / 15\right)$ such as $\left\langle\delta u(r)^{2}\right\rangle=U^{2} b_{l l}^{0}\left(x_{0}\right)$ with $x_{0}=r / L, \quad$ and $\left\langle\delta u(r)^{2}\right\rangle=U^{2} R_{L}^{-1 / 2} b_{l l}^{i}\left(x_{i}\right)$ with $x_{i}=r / \eta$ $=R_{L}^{3 / 4} x_{0}$.

Now, remarking that the von Karman equation in the outer (respectively, inner) variables has $R_{L}^{-1}$ (respectively, $R_{L}^{-1 / 2}$ ) as small parameter, he writes $b_{l l}^{0}=b_{l l}^{0,1}+R_{L}^{-1} b_{l l}^{0,2}$ $+\cdots$ and $b_{l l}^{i}=b_{l l}^{i, 1}+R_{L}^{-1 / 2} b_{l l}^{i, 2}+\cdots$.

But, as remarked by Barenblatt, ${ }^{18}$ incomplete selfsimilarity can result in the absence of limit for infinite $R_{L}$ for one of these functions. The general case writes $b_{l l}^{i}$ $=R_{L}^{-\delta} b_{l l}^{i, 1}+\cdots$.

Then, Eq. (27) of Lundgren, which ensures the matching of the two expressions, becomes $U^{2} R_{L}^{-1 / 2} R_{L}^{-\delta} b_{l l}^{i, 1}\left(x_{0} R_{L}^{3 / 4}\right)$ $=U^{2} b_{l l}^{0,1}\left(x_{0}\right)$ and thus $b_{l l}^{0,1}=C_{K} x_{0}^{2(1+2 \delta) / 3}$ and $b_{l l}^{i, 1}$ $=C_{K} x_{i}^{2(1+2 \delta) / 3}$.

By the comparison between these two latest equations and the result of intermittency studies, the value of $\delta$ should be of order $\delta \simeq 0.02$. The two exponents $\gamma_{0}$ and $\gamma_{i}$ (which are the limit slopes in the log-similar diagram) are $\gamma_{0}=2 / 3$ $+4 \delta / 3$ and $\gamma_{i}=-4 / 3+4 \delta / 3$. The minimum occurs at $\beta \ln (r / \lambda)=\delta / 2$ and its value is $-2 / 3-\delta$. The very small value of $\delta$ explains why the difference with the Lundgren prediction is not visible.

In summary, the present experimental data confirm the very slow convergence of $S_{3}(r)$ towards an asymptotic re- gime, whatever the flow type and/or the Reynolds number. Also, the pertinent scale of $\left[S_{3}(r)\right]_{\max }$ seems to be close to the Taylor microscale but with a different Reynolds scaling. From an experimental point of view, the log-similarity plot suits to account for the detailed behavior of $S_{3}(r)$ in the whole inertial range when the Reynolds number tends to infinity. The question of intermittency is not relevant here and must be studied by other means.

${ }^{1} \mathrm{U}$. Frisch, Turbulence: The Legacy of A. N. Kolmogorov (Cambridge University Press, Cambridge, 1995).

${ }^{2}$ A. S. Monin and A. M. Yaglom, Statistical Fluid Mechanics (MIT Press, Cambridge, MA, 1975).

${ }^{3}$ K. R. Sreenivasan and R. A. Antonia, "The phenomenology of small-scale turbulence," Annu. Rev. Fluid Mech. 29, 435 (1997).

${ }^{4}$ M. Nelkin, "Universality and scaling in fully developed turbulence," Adv. Phys. 43, 143 (1994).

${ }^{5}$ F. Moisy, P. Tabeling, and H. Willaime, "Kolmogorov equation in fully developed turbulence," Phys. Rev. Lett. 82, 3994 (1999).

${ }^{6} \mathrm{~L}$. Mydlarski and Z. Warhaft, "On the onset of high Reynolds-number grid-generated wind tunnel turbulence," J. Fluid Mech. 320, 331 (1996).

${ }^{7}$ B. Pearson and R. A. Antonia, "Reynolds-number dependence of turbulent velocity and pressure increment," J. Fluid Mech. 444, 343 (2001).

${ }^{8} \mathrm{~J}$. Qian, "Inertial range and the finite Reynolds number effect of turbulence," Phys. Rev. E 55, 337 (1997).

${ }^{9}$ J. Qian, "Slow decay of the finite Reynolds number effect of turbulence," Phys. Rev. E 60, 3409 (1999).

${ }^{10} \mathrm{~T}$. S. Lundgren, "Kolmogorov two-thirds law by matched asymptotic expansion," Phys. Fluids 14, 638 (2002).

${ }^{11}$ T. S. Lundgren, "Kolmogorov turbulence by matched asymptotic expansion," Phys. Fluids 15, 1074 (2003).

${ }^{12}$ Y. Malécot, C. Auriault, H. Kahalerras, Y. Gagne, O. Chanal, B. Chabaud, and B. Castaing, "A statistical estimator of turbulence intermittency in physical and numerical experiments," Eur. Phys. J. B 16, 549 (2000).

${ }^{13}$ J. F. Pinton and R. Labbe, "Corrections to the Taylor hypothesis in swirling flows," J. Phys. II 54, 1461 (1994).

${ }^{14}$ E. Gledzer, "On the Taylor hypothesis corrections for measured energy spectra in turbulence," Physica D 104, 163 (1997).

${ }^{15}$ B. Dhruva, Y. Tsuji, and K. R. Sreenivasan, "Transverse structure functions in high Reynolds number turbulence," Phys. Rev. E 56, 4928 (1997).

${ }^{16}$ L. Danaila, F. Anselmet, T. Zhou, and R. A. Antonia, "A generalisation of Yaglom's equation which accounts for the large-scale forcing in heated decaying turbulence," J. Fluid Mech. 391, 359 (1999).

${ }^{17}$ O. Chanal, B. Chabaud, B. Castaing, and B. Hebral, "Intermittency in a low temperature gaseous helium jet," Eur. Phys. J.: Appl. Phys. 17, 309 (2000).

${ }^{18}$ G. Barenblatt, "Scaling laws for fully developed turbulent shear flows," J. Fluid Mech. 248, 513 (1993). 